\title{
Das Jahr
}

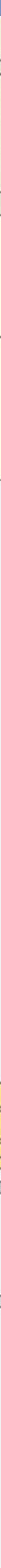

1 Was siehst du? Sprich.

2 Höre und sprich nach. (2) (잉

\section{Handy}


Das Jahr

3a Sprich nach und fahre mit dem Finger nach. (3) () (16)
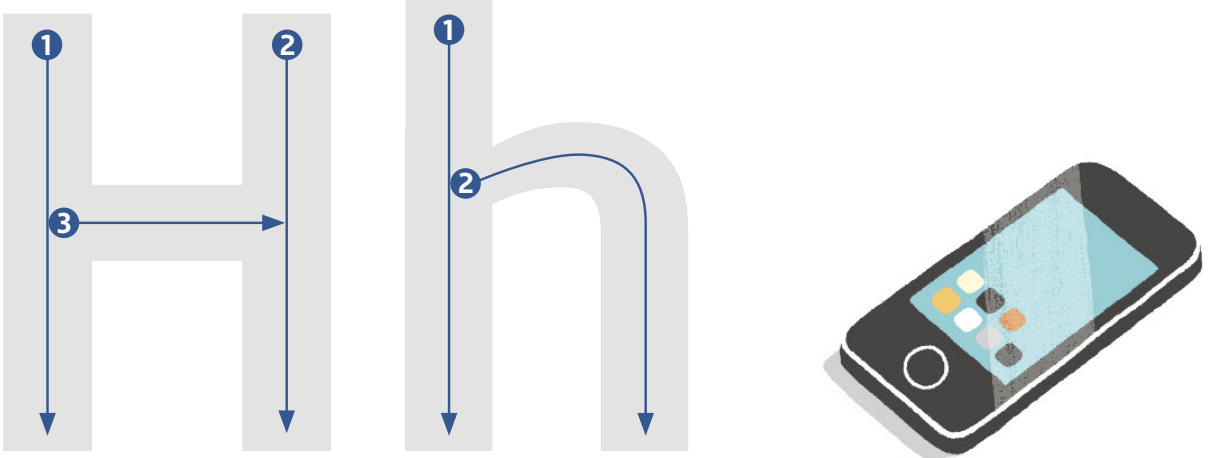

3b Schreibe.

$\mathrm{H}$

h

$\mathrm{H}$

h

$\mathrm{Hh}$

das Handy

3c Schreibe die Artikel.

Handy

Herbst

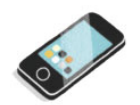

Haus

Hand
Hof

Hose

Hals

Hund 0 
4 In welchem Wort hörst du H h? Kreuze an. (2) (4)
$\mathbf{0} \square$
$2 \square$
3
$\boldsymbol{4} \square$
(5) $\square$
$\boldsymbol{0} \square$

5a Lies. 요

\begin{tabular}{|l|l|l|}
\hline $\begin{array}{l}\text { 1. Monat } \\
\text { 2. Monat }\end{array}$ & $\begin{array}{l}\text { der Januar } \\
\text { der Februar }\end{array}$ & der Winter \\
\hline $\begin{array}{l}\text { 3. Monat } \\
\text { 4. Monat } \\
\text { 5. Monat }\end{array}$ & $\begin{array}{l}\text { der März } \\
\text { der April } \\
\text { der Mai }\end{array}$ & der Frühling \\
\hline $\begin{array}{l}\text { 6. Monat } \\
\text { 7. Monat } \\
\text { 8. Monat }\end{array}$ & $\begin{array}{l}\text { der Juni } \\
\text { der Juli } \\
\text { der August }\end{array}$ & der Sommer \\
\hline $\begin{array}{l}\text { 9. Monat } \\
\text { 11. Monat }\end{array}$ & $\begin{array}{l}\text { der September } \\
\text { der Oktober } \\
\text { der November }\end{array}$ & der Herbst \\
\hline 12. Monat & der Dezember & der Winter \\
\hline
\end{tabular}

5b Schreibe die Monate.

\begin{tabular}{|c|c|c|c|}
\hline der Frühling & der Winter & der Herbst & der Sommer \\
\hline der März & & & \\
\hline & & & \\
\hline & & & \\
\hline
\end{tabular}


Das Jahr

6 Höre und sprich nach . (9) (1) 18

Januar $\bullet . \quad$ Juli $\bullet$

Februar $\bullet$ August . $\bullet$

März • September $\bullet^{\bullet}$.

April $\bullet^{\bullet} \quad$ Oktober $\bullet^{\bullet}$.

Mai $\bullet \quad$ November $\bullet \bullet$

Juni $\bullet \quad$ Dezember $\bullet \bullet$

7 Höre und schreibe. (2) (?)

Wann ist Winter?

Winter ist im Dezember. Januar und Februar.

Wann ist Frühling?

Wann ist Sommer?

Wann ist Herbst? 
8a Verbinde.

der Frühling

der Sommer

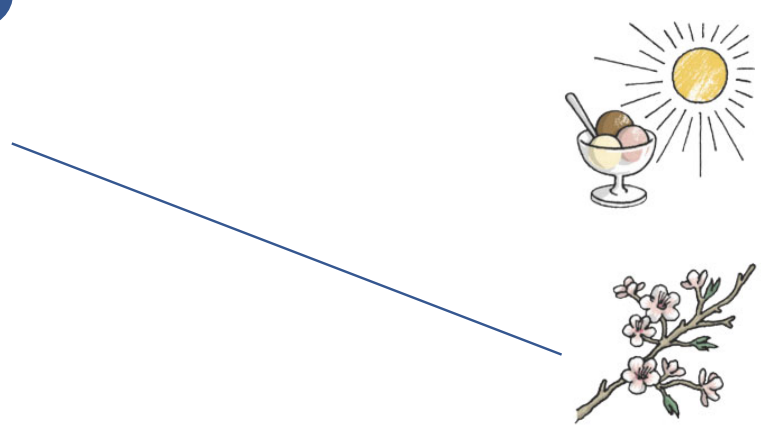

der Herbst

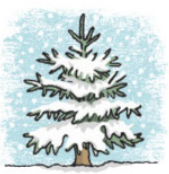

der Winter

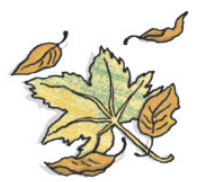

8b Höre das Lied und schreibe. (2) (?)

Es war eine Mutter, die hatte vier Kinder.

Den_, den

den__ und den

Der_bringt Blumen,

der_bringt Klee.

Der_bringt die Trauben,

der__ den Schnee.

8c Höre das Lied und singe mit. (2) (1) 2 


\section{Das Jahr}

9a Welches H sprichst du? Lies den Text.

Hallo. Mein Name ist Herr Hirata.

Ich bin Lehrer.

Ich schreibe hier alle Wörter mit $\mathrm{H}$ h:

Wir sprechen $\mathrm{H}$ h nur am Wortanfang und Silbenanfang. die Hand, die Uhr, die Hose, die Zahl, das Handy, das Ohr, das Haus, der Herbst, der Schuh.

Tschüss und auf Wiedersehen.

9b Markiere $\mathrm{H}$ h.

10 Gestalte deinen Kalender.

\begin{tabular}{|l|r|l|l|}
\hline Januar & & Juli & \\
\hline Februar & & August & \\
\hline März & & September & \\
\hline April & & Oktober & \\
\hline Mai & & November & \\
\hline Juni & & Dezember & \\
\hline
\end{tabular}


11a Höre und lies mit. (9) (1) 22 영

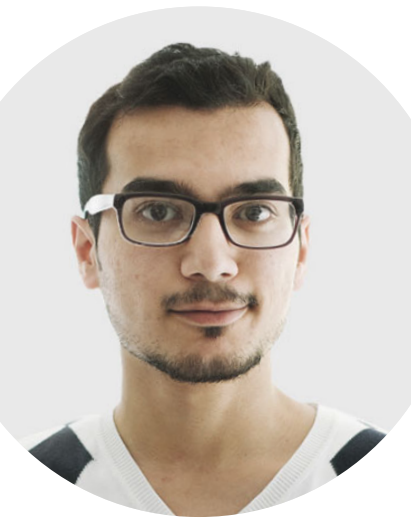

Hallo. Ich bin Tarek Haoud. Meine Handynummer ist $01151 / 14851346$.

Wie ist deine Handynummer? Tschüss.

11b Meine Aufgabe für dich. Schreibe das Wort oder male.
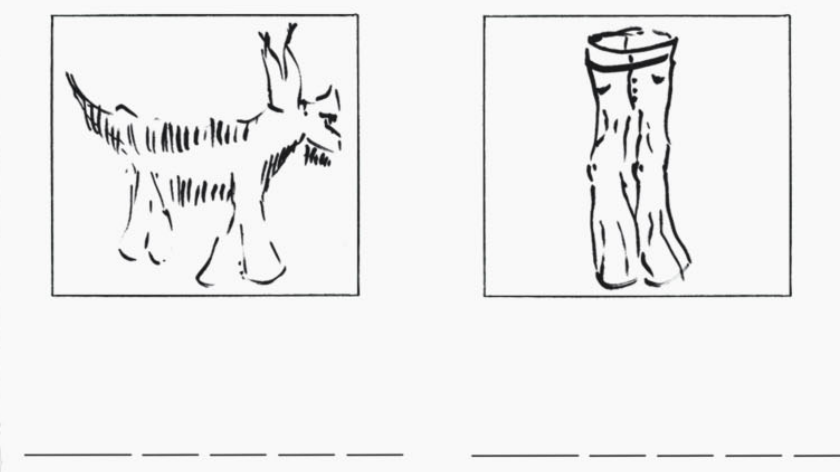

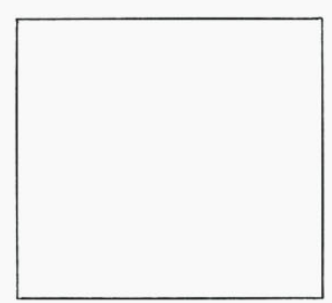

das Haus

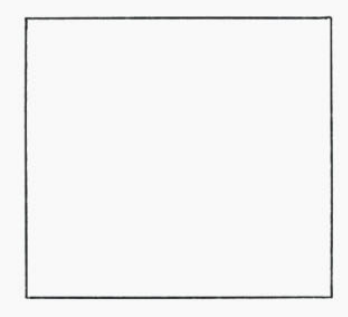

die Hand

11c Schreibe eine eigene Aufgabe. 
$\star$ Zeichne die Silbenbögen ein und lies.

Ich habe Hunger.

Ich esse Salat.

Ich liebe Schokolade!

Ich brauche ein Glas.
Ich habe Durst.

Ich trinke Wasser.

Guten Appetit!

Ich möchte eine Cola.

$\star \star$ Schreibe den unbestimmten Artikel.

Hand

Monat

Handy

Haus
Jahr

Tag

Herbst

Hals
Hose

Uhr

Hund

Ohr

$\star \star \star$ Schreibe.

\begin{tabular}{|l|l|l|l|}
\hline der Wi_ter & der Frü_ling & der So__er & der_erbst \\
\hline D__zember & Mä_z & J_ni & S_ptember \\
\hline J_n_ar & Ap_i__ & J_l_ & _kt_ber \\
\hline _ebr_ar & _ai & _ug_st & $\mathrm{N} \_$vember \\
\hline
\end{tabular}


$\star$ Verbinde und schreibe.

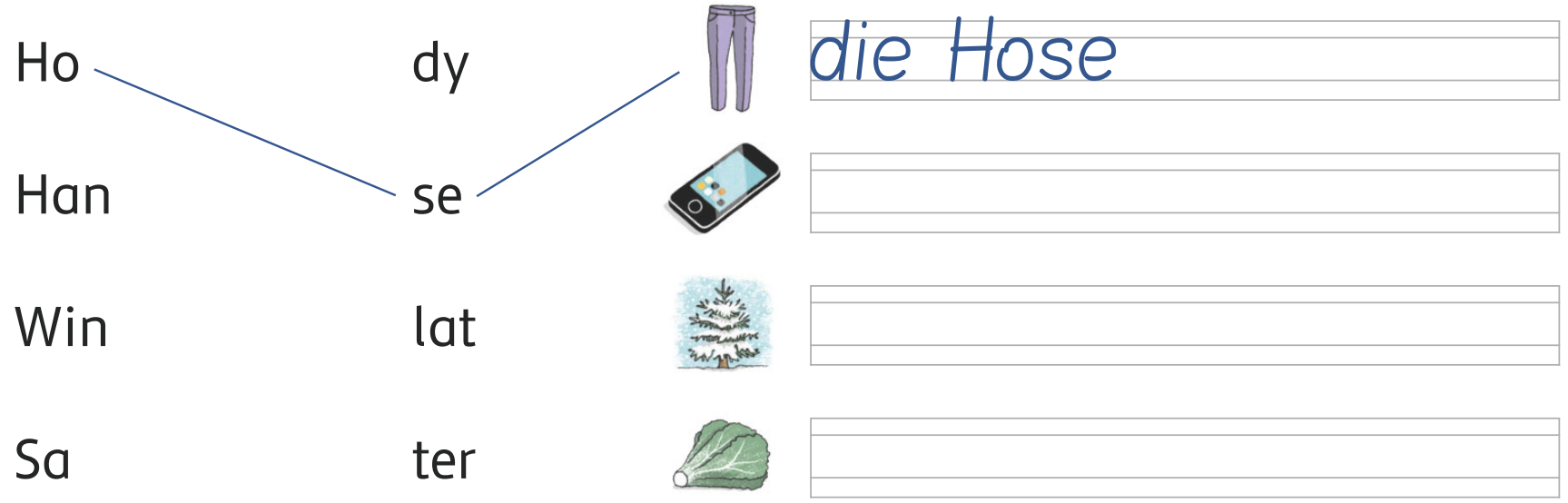

$\star \star$ Welche Wörter schreibt man groß? Markiere und schreibe. heißen hier hallo haus haben handy heute

$\star \star \star$ Lies und schreibe.

Am $M \ldots$ ntag les_ ich ein $B \ldots$ ch.

Am Dienst_g schr_ be ich in Wort.

Am Mittw_ch le ne ich Deut

Am Donner_ tag $\mathrm{m}$ _che ich Spo_ $\mathrm{t}$.

Am Fr__tag esse _ ch Supp_..

Am Sa _ stag tr_ nke ich__ilch. 
圈 Mein Wortschatz

Schreibe.

㗪 der Frühling

* der Sommer

触 der Herbst

der Winter

der Januar

der Februar

der März

der April

der Mai

der Juni

der Juli

der August

der September

der Oktober

der November

der Dezember 
Sortiere die Monatskarten zu den Jahreszeiten.

der Januar

der Februar

der März

der April

der Mai

der Juni

der Juli

der August der September

der Oktober

der November

der Dezember

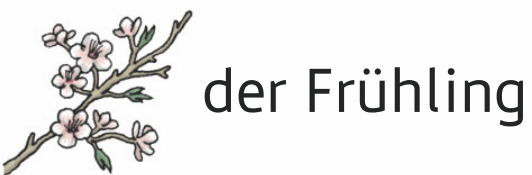

der Sommer

der Herbst

zols der Herbst

der Winter 
Open Access Dieses Kapitel wird unter der Creative Commons Namensnennung - Nicht kommerziell - Keine Bearbeitung 4.0 International Lizenz (http://creativecommons.org/licenses/by-nc-nd/4.0/deed.de) veröffentlicht, welche die nicht-kommerzielle Nutzung, Vervielfältigung, Verbreitung und Wiedergabe in jeglichem Medium und Format erlaubt, sofern Sie den/die ursprünglichen Autor(en) und die Quelle ordnungsgemäß nennen, einen Link zur Creative Commons Lizenz beifügen und angeben, ob Änderungen vorgenommen wurden. Die Lizenz gibt Ihnen nicht das Recht, bearbeitete oder sonst wie umgestaltete Fassungen dieses Werkes zu verbreiten oder öffentlich wiederzugeben.

Die in diesem Kapitel enthaltenen Bilder und sonstiges Drittmaterial unterliegen ebenfalls der genannten Creative Commons Lizenz, sofern sich aus der Abbildungslegende nichts anderes ergibt. Sofern das betreffende Material nicht unter der genannten Creative Commons Lizenz steht und die betreffende Handlung nicht nach gesetzlichen Vorschriften erlaubt ist, ist auch für die oben aufgeführten nicht-kommerziellen Weiterverwendungen des Materials die Einwilligung des jeweiligen Rechteinhabers einzuholen. 